\title{
Commentary: Autopsy Validation of 123I-FP-CIT Dopaminergic Neuroimaging for the Diagnosis of DLB
}

\author{
Curtis Osborne*, John Paul Taylor, Alan J. Thomas
}

Institute of Neuroscience, Biomedical Research Building, Campus for Ageing and Vitality, Newcastle University, Newcastle upon Tyne, UK

\section{Article Info}

\section{Article Notes}

Received: August 07, 2018

Accepted: August 23, 2018

\section{${ }^{*}$ Correspondence}

Dr. Curtis Osborne, Institute of Neuroscience, Biomedical Research Building, Campus for Ageing and Vitality, Newcastle University, Newcastle upon Tyne, UK; E-mail: Curtis.Osborne@ ntw.nhs.uk.

(c) 2018 Osborne C. This article is distributed under the terms of the Creative Commons Attribution 4.0 International License

\section{Introduction}

Dementia with Lewy bodies is the second most common cause of dementia after Alzheimer's disease. One of the key features of the disease is the loss of dopaminergic neurons in the nigrostriatal pathway. Their loss is associated with the presence of a-synuclein aggregates, which is one of the hallmarks of Lewy Body Disease (LBD) ${ }^{1}$. Currently the gold standard for diagnosis of alpha synucleinopathies, including DLB, is autopsy. However, neuroimaging provides in-vivo evidence of underlying neuropathological features of DLB, in line with the recent NIA-AA Framework for $\mathrm{AD}^{2}$. This paper focuses on a study by Thomas et al., 2017 which validates 123I-FP-CIT neuroimaging against autopsy. This paper will highlight the importance of combining clinical acumen and neuroimaging in DLB diagnosis to improve clinical confidence in DLB diagnosis ${ }^{3}$.

123I-FP-CIT is a radioactive striatal dopamine transporter binding agent which can be visualized using SPECT brain imaging, which in turn allows for a quantitative measure and analysis of spatial distribution of the dopamine transporters. There have been several recent studies which have evaluated 123I-FP-CIT against neuropathology in DLB-6. One examined 20 patients (eight with DLB) and found that 123I-FP-CIT had a sensitivity of $88 \%$ and specificity of $100 \%{ }^{4}$. Another investigated neuronal loss and pathology in 23 cases (seven with DLB), reporting an association between neuronal density in the substantia nigra and reduced uptake on 123I-FP-CIT ${ }^{5}$. A more recent study by Jung et al 2018 used $^{123}$ I-FP-CIT SPECT to investigate striatal uptake and found an abnormal ${ }^{123}$ I-FP-CIT SPECT was strongly associated with the presence of underlying Lewy body disease pathology at autopsy ${ }^{6}$.

Thomas et al., $2017^{3}$ reported the largest study to date using a sample of 55 patients (33 with DLB and 22 with AD) who had come to autopsy at Newcastle Brain Tissue Resource and who had had FP-CIT imaging during life. The study aimed to validate the use of 123I-FP-CIT by comparing its diagnostic accuracy in differentiating $A D$ and $D^{2} B^{3}$ and by comparing the diagnostic accuracy of FP-CIT with in vital clinical diagnosis. The average age in the DLB and AD groups was in the mid70 s, typical of DLB studies, and there was no significant difference in the ratio of males and females between the two groups. The AD patients however were significantly older at age of death with an average age of 86.0 compared with that of 78.8 in the DLB group, which is consistent with evidence of higher mortality in DLB than $\mathrm{AD}^{7}$.

The main limitation of this study is that the clinical diagnoses of dementia were made using the neurotic Braak staging and CERAD 
criteria for AD and the 1996 Newcastle -McKeith criteria for DLB which limits diagnostic accuracy when compared to present practice; the 1996 McKeith clinical diagnostic criteria are demonstrably less robust and lacked the sensitivity compared to 2005 consensus criteria for the diagnosis and management of $\mathrm{DLB}^{1}$. However despite this limitation the DLB cohort in the study demonstrated the core symptoms of DLB and were similar to cohorts in other DLB studies carried out during the same period.

Each participant underwent a clinical assessment, a 123I-FP-CIT scan and was followed up until death with subsequent autopsy. As expected in a large autopsy series, some cases had major pathology identified at autopsy which was neither AD nor LB disease (one of the DLB and two of the AD patients had frontotemporal lobar degeneration (FTLD) and one with DLB had cortico-basal degeneration (CBD). Since the aim of the study was to identify the accuracy of FP-CIT in facilitating the correct diagnosis of DLB vs other dementias these cases were placed in the non-LB pathology category. The existence of such patients demonstrates the difficulty in diagnosis of DLB and AD clinically, especially prior to the more recent 2005 criteria, which has helped to improve diagnosis of DLB $^{9}$. Neuropathologically those with mixed DLB and AD were incorporated into the LB group because they had evidence of clinically important (limbic and/or neocortical) LB disease, despite their concurrent high grade (Braak >4) AD neuropathological change.

\section{I-FP-CIT Findings}

The final groups by autopsy were 30 for the LB cohort (pure DLB and mixed AD+DLB) and the non-LBD cohort consisted of 25 participants $(21=\mathrm{AD}, 3=$ FTLD and one CBD). Of the 30 confirmed LBD cases at autopsy, 24 were found to have abnormal 123I-FP-CIT imaging. Of the 25 non-LBD cases 23 had normal 123I-FP-CIT imaging. Thus, the study by Thomas et al. 2017 found that 123I-FP-CIT imaging had a sensitivity of $80 \%$ and a specificity of $92 \%$. The overall diagnostic accuracy of identifying of LBD vs non-LBD using 123I-FP-CIT imaging was $86 \%$. This is consistent with another recent autopsy study by Jung et al., 2018 who demonstrated the sensitivity and specificity of abnormal ${ }^{123}$ I-FP-CIT SPECT with reduced striatal uptake on visual inspection for predicting Lewy body disease to be $91.7 \%$ and $83.3 \%$, respectively ${ }^{6}$.

\section{Clinical Diagnostics}

The imaging result was compared to the accuracy of clinical diagnosis. Clinical diagnoses were made by clinicians blinded to the 123I-FP-CIT results and applying standard research criteria for AD dementia and DLB. It was found that of the 30 autopsy confirmed cases of LB disease, 26 were diagnosed with DLB clinically and four were diagnosed with $\mathrm{AD}$ resulting in a sensitivity of $87 \%$.
Of the 25 non-LBD cases 18 were diagnosed as having AD and seven with DLB (specificity of $72 \%$ ). The overall diagnostic accuracy of identifying of LBD vs non-LBD using 1996 clinical criteria alone was therefore $79 \%$, which is $7 \%$ less than FP-CIT, but further work would be required to determine if this disparity remains with the updated 2017 consensus criteria ${ }^{10}$.

\section{Clinical Lessons from False Positive and False Negative Cases}

Analysis of the 123I-FP-CIT scans found two false positives (with reduction in striatal uptake) and six false negatives (withnoreduction in striatal uptake) in the sample. One false positive had clinical evidence of Parkinsonism and cognitive fluctuations but neuropathology was consistent with FTLD. The other had three core DLB symptoms but autopsy assessment demonstrated AD neuropathologic change. However, in both of these cases there was evidence of abnormal neuropathology within the striatum which may have accounted for the DLB-like symptoms found clinically as well as the reduced 123I-FP-CIT uptake. This reminds us that although FP-CIT is a very accurate biomarker it is not a direct marker of synuclein or LB pathology. Other diseases, such as FTLD, which damage the substantia nigra neurons can cause abnormal FP-CIT scans ${ }^{11}$. This is important as both Logopenic Progressive Aphasia (LPA) and semantic dementia overlap clinically with AD but have underlying FTLD pathology ${ }^{12}$. Thus, clinical acumen is essential as a positive FP-CIT is not itself diagnostic of DLB and would be unable to differentiate between other alphasynucleinopathies. This includes progressive supranuclear palsy and multi-system atrophy which present with parkinsonism and demonstrate reduced 123I-FP-CIT uptake similar to that of DLB.

of the six false-negative cases, three had a clinical baseline clinical diagnosis of $\mathrm{AD}$ and mixed $\mathrm{LBD}+\mathrm{AD}$ pathology at autopsy. These would appear to be cases in whom the AD pathology masked the presence of LB disease and this fits the wider literature showing that multiple pathologies are commonly present in those diagnosed with clinical dementia ${ }^{13}$. These additionally highlight the limitations of FPCIT which focusses on the striata and thus cannot identify LB pathology elsewhere, whereas other biomarkers, e.g. 123I-metaiodobenzylguanidine (MIBG) cardiac imaging, might do so in such cases. ${ }^{2}$. The other three false-negative cases all met clinical consensus criteria for probable DLB at the time of 123I-FP-CIT, having complex visual hallucinations and marked cognitive fluctuations, and neuropathologically fulfilled the criteria for DLB. These cases are important because they demonstrate that a negative (normal) FP-CIT scan does occur in people with clinically and pathologically confirmed LB disease. In such people it appears that the LB disease predominantly involves higher cortical areas to cause dementia and 
also visual hallucinations and cognitive fluctuations. But LB involvement of the brainstem and specifically the substantia nigra was minimal or absent and so the FP-CIT scan was normal. Thus, again in these cases if the clinical suspicion of DLB remains high MIBG cardiac imaging could be utilized as it has been demonstrated that although 123I-FP-CIT is superior at detecting LB disease with evidence of Parkinsonism whilst MIBG cardiac imaging has high sensitivity in suggesting the presence of LB disease, indirectly via noradrenergic dysfunction, in those who have REM sleep behavior disorder ${ }^{14}$.

\section{Conclusion}

123I-FP-CIT imaging has very good diagnostic accuracy (86\%) with good sensitivity (80\%) and specificity (92\%) for distinguishing DLB from AD and this has been validated against autopsy diagnosis. This is consistent with previous work in which 123-FP-CIT was compared to clinical diagnosis by a panel of experts ${ }^{15}$. The use of FP-CIT is also supported in NICE guidance in preference to MIBG. This is primarily due to the more robust evidence base for FPCIT produced by validation in studies such as Thomas et al., 2017 and Jung et al., 2018. However, there is a still an important role for MIBG for investigating DLB, such as when FP-CIT is negative and clinical suspicion remains high, or when there is a suggestion that another disease which can affect the striatum and cause abnormal FP-CIT (false positive) might be present, e.g. FTD, or when FP-CIT is unavailable ${ }^{16}$. Thus, the role of FP-CIT imaging currently would be when a clinician has clinical suspicion but diagnostic uncertainty about a diagnosis of DLB. Further work is required to investigate the usefulness of FP-CIT in detecting early DLB with findings to date indicating FP-CIT has high specify for detecting progression to DLB in mild cognitive impairment cohorts ${ }^{17}$. Its use here would provide an opportunity for improved early diagnosis and the possibility of introducing disease modifying agents before functional impairment arises.

\section{Acknowledgment}

The research was supported by Alzheimer's Research UK and the National Institute for Health Research Newcastle Biomedical Centre based at Newcastle upon Tyne Hospitals NHS Foundation Trust and Newcastle University. The views expressed are those of the authors and not necessarily those of the NHS, the NIHR or the Department of Health.

\section{References}

1. McKeith IG, Dickson DW, Lowe J, et al. Diagnosis and management of dementia with Lewy bodies: third report of the DLB consortium. Neurology. 2005; 65: $1863-1872$.
2. Jack CR, Bennett DA, Blennow K, et al. NIA-AA Research Framework: Toward a biological definition of Alzheimer's disease. Alzheimer's \& dementia the journal of the Alzheimer's Association. 2018; 14(4): 535-562.

3. Thomas AJ, Attems J, Colloby SJ, et al. Autopsy validation of ${ }^{123}$ I-FPCIT dopaminergic neuroimaging for the diagnosis of DLB. Neurology. 2017; 88(3): 276-283.

4. Walker Z, Jaros E, Walker RW, et al. Dementia with Lewy bodies: a comparison of clinical diagnosis, FP-CIT single photon emission computed tomography imaging and autopsy. J Neurol Neurosurg Psychiatry. 2007; 78: 1176 -1181.

5. Colloby SJ, McParland S, O 'Brien JT, et al. Neuropathological correlates of dopaminergic imaging in Alzheimer 's disease and Lewy body dementias. Brain. 2012; 135(pt 9): 2798 -2808.

6. Jung Y, Jordan LG, Lowe VJ, et al. Clinicopathological and ${ }^{123}$ I-FP-CIT SPECT correlations in patients with dementia. Annals of Clinical and Translational Neurology. 2018; 5(3): 376-381.

7. Garcia-Ptacek S, Farahmand B, Kareholt I, et al. Mortality risk after dementia diagnosis by dementia type and underlying factors: a cohort of 15,209 patients based on the Swedish Dementia Registry. J Alzheimers Dis 2014; 41: 467-477.

8. Skogseth R, Hortobágyi T, Soennesyn H, et al. Accuracy of Clinical Diagnosis of Dementia with Lewy Bodies versus Neuropathology. J Alzheimers Dis. 2017; 59(4): 1139-1152

9. McKeith IG, Boeve BF, Dickson DW, et al. Diagnosis and management of dementia with Lewy bodies: Fourth consensus report of the DLB Consortium. Neurology. 2017; 89(1): 88-100.

10. McKeith IG, Boeve BF, Dickson DW, et al. Diagnosis and management of dementia with Lewy bodies: Fourth consensus report of the DLB Consortium. Neurology. 2017; 89(1): 88-100.

11. Morgan S, Kemp P, Booij J, et al. Differentiation of frontotemporal dementia from dementia with Lewy bodies using FP-CIT SPECT. J Neurol Neurosurg Psychiatry. 2012; 83: 1063-1070.

12. Goedert M, Ghetti B, Spillantini MG. Frontotemporal Dementia: Implications for Understanding Alzheimer Disease. Cold Spring Harbor Perspectives in Medicine. 2012; 2(2): a006254.

13. Jellinger KA, Attems J. Prevalence and pathology of dementia with Lewy bodies in the oldest old: a comparison with other dementing disorders. Dement Geriatr Cogn Disord. 2011; 31: 309-316

14. Shimizu S, Hirao K, Kanetaka $\mathrm{H}$, et al. Utility of the combination of DAT SPECT and MIBG myocardial scintigraphy in differentiating dementia with Lewy bodies from Alzheimer's disease. European Journal of Nuclear Medicine and Molecular Imaging. 2016; 43: 184-192.

15. 0 'Brien JT, Oertel WH, McKeith IG, et al. Is ioflupane I123 injection diagnostically effective in patients with movement disorders and dementia? Pooled analysis of four clinical trials. BMJ Open. 2014; 4: e005122

16. National Institute for Health and Care Excellence (2018) Dementia: assessment, management and support for people living with dementia and their carers (NICE Guideline 97) Available at:https://www. nice.org.uk/guidance/ng97/chapter/Recommendations\#diagnosis [Accessed 15/07/2018].

17. Thomas AJ, Donaghy P, Roberts G, et al. Diagnostic accuracy of dopaminergic imaging in prodromal dementia with Lewy bodies. Psychol Med. 2018; 1-7. 